\title{
Application of the Spatial Distribution Function to Colloidal Ordering
}

\author{
Niamh Mac Fhionnlaoich, Runzhang Qi, and Stefan Guldin* \\ University College London, Department of Chemical Engineering, Torrington Place, WC1E \\ YJE, London \\ E-mail: s.guldin@ucl.ac.uk
}

\begin{abstract}
$2 \mathrm{D}$ colloidal assembly is a vital process in the fabrication of nanostructured devices and remains of widespread interest in fundamental research. Characterising the ordering is crucial to develop an understanding of the driving forces behind the assembly and to optimise processing conditions. Image analysis offers a direct evaluation pathway, typically via the radial distribution function or the 2D-fast Fourier transform. Both methods have inherent limitations; the former provides no angular dependence while the latter is challenged when confronted with imperfection on the mean size, spacing and coverage of the building blocks. Here, we introduce the 2D spatial distribution function (SDF) as an alternative pathway to evaluate colloidal ordering. We benchmark the method in case studies of prominent examples and provide a tool-kit for implementation, either as imageJ plugin or standalone software. Application and interpretation is straightforward and particularly powerful to analyse and compare colloidal assemblies with limited order.
\end{abstract}




\section{Introduction}

Evaluating colloidal ordering is of widespread importance to determine crucial process conditions, which in turn allows for a greater degree of control over the materials assembly. ${ }^{1}$ Important parameters include the structural symmetry, inter-particle spacing, and the distance over which order persists. While small angle X-ray scattering and laser diffraction provide powerful routes for evaluation, the most common techniques are predominantly microscopy-based, such as transmission electron microscopy (TEM), scanning electron microscopy (SEM), atomic force microscopy (AFM), and optical microscopy. Among a plethora of available image analysis routines, ${ }^{2-4}$ two of the most common ones are the $2 \mathrm{D}$ fast Fourier transform (2D FFT) and the radial distribution function (RDF).

The 2D-FFT represents an image of the assembly in the frequency domain. This process converts patterns within an image into frequencies, which highlights information about the structural symmetry and the extent of order. A high degree of order translates into distinct spatial frequencies in the Fourier transform, and thus well-defined peaks can be found in the frequency image. With compartmentalization and translational rotation of individual domains, the peaks blur into rings; as the inter-particle spacing decreases in coherence, the peaks render less profound and eventually vanish. ${ }^{1,5}$ The effectiveness of the 2D FFT (and the associated power spectral density) to gain a qualitative and quantitative understanding of the colloidal ordering has been demonstrated in numerous studies, e.g. to understand the principles and dynamics of the assembly process, ${ }^{6-8}$ evaluate the symmetry and orderliness of building block arrangement, ${ }^{8,9}$ and determine the structure of binary superlattices. ${ }^{10-12}$

The RDF, or pair correlation function, represents the density of particles as a function of distance from a reference particle. ${ }^{13}$ The resulting plot shows peaks at prescribed distances depending on the type of packing observed. ${ }^{14}$ Many studies have used the RDF for structure determination (based on the positions of the peaks) and evaluation of the extend of ordering (via the number and broadness of the peaks). ${ }^{15-18}$ While for less well-ordered assemblies the footprint of the underlying structure may not directly depictable from the RDF, it provides a 
robust pathway to determine inter-particle spacing and assess long vs. short range ordering. ${ }^{19}$

Although both techniques are undoubtedly useful, each has intrinsic limitations. The 2DFFT does not show the results in real space and additional processing is required to extract the inter-particle spacing. Furthermore, as disorder in the system increases, evidence of the extent of order is quickly lost. The 2D-FFT is a fully reversible analytic process; its inverse will accurately reproduce the original image of the sample used. As such, the 2D-FFT must store additional information such as particle size, gradient, etc. This information is not useful in determining the spatial arrangement and may negatively impact the analysis. The $\mathrm{RDF}$, in contrast, excludes this information and focuses solely on the positional data. It retains information about the extent of ordering even in noisy systems, but it lacks any directional information and extracting structural information can be difficult or impossible

for imperfect assemblies. ${ }^{20}$ Thus, both techniques often need to be used in combination to evaluate the ordering within a colloidal assembly. ${ }^{21}$

The 2D spatial distribution function (SDF) represents an alternative that combines aspects of 2D-FFT and RDF. Variations of this method have been used in the past to elucidate local atomic densities, where it provides the angular dependency in contrast to the RDF and manages with the disorder arising from natural fluctuations in bond length and angle. ${ }^{22-24}$

Here, we introduce the use of the $2 \mathrm{D}$ spatial distribution function (SDF) in evaluating colloidal ordering. Various examples of symmetry and order serve as case studies to benchmark the SDF to the more widely established 2D FFT and RDF. Importantly, we provide both an ImageJ plugin and a standalone software for the implementation of the SDF and provide guidelines for interpretation.

\section{Experimental}

ImageJ was used to determine the $(\mathrm{x}, \mathrm{y})$ positions of each particle in a given assembly. Further details on this step can be found in the SI (Figure S1). This positional array was then fed 

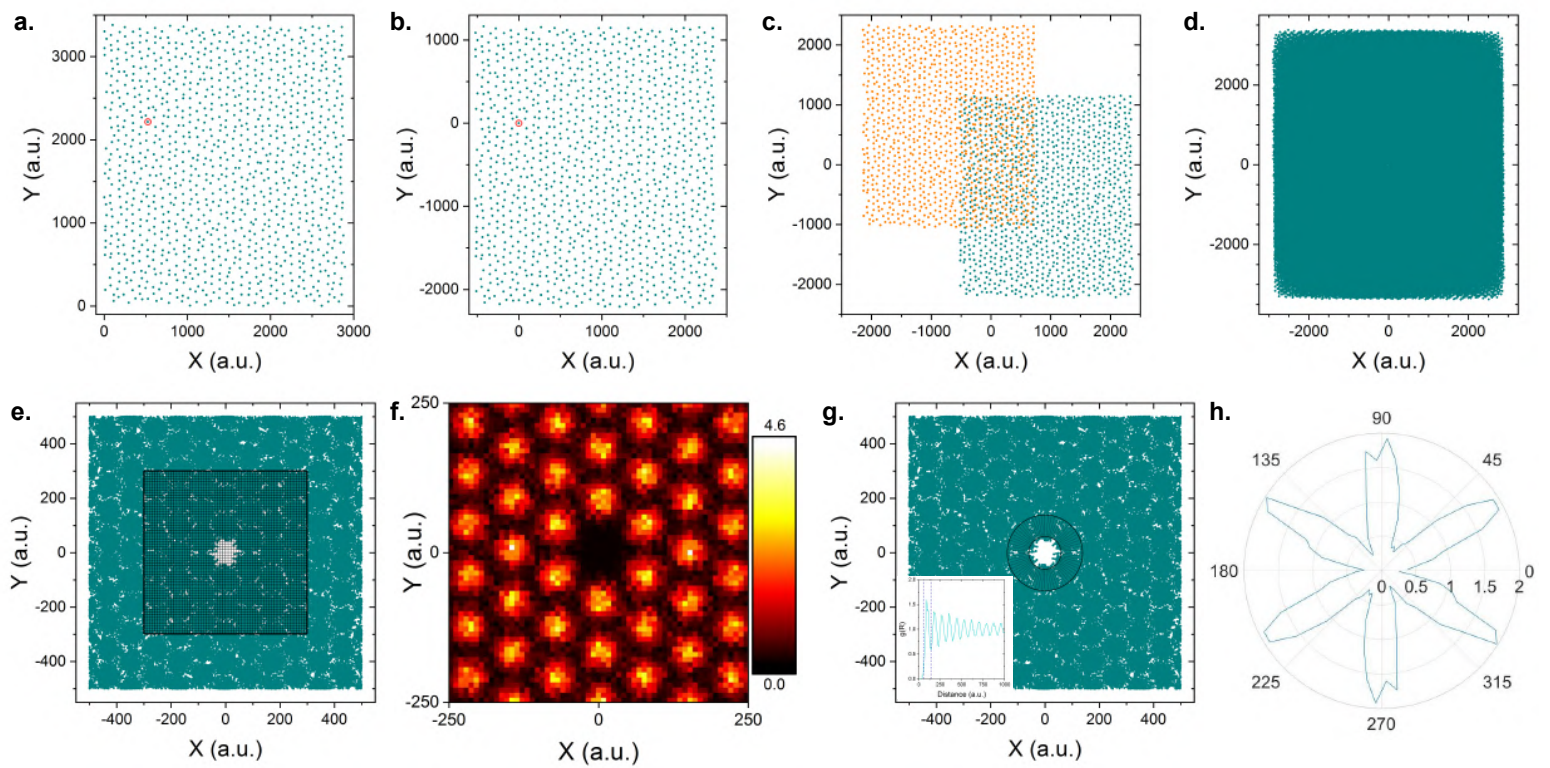

Figure 1: Construction of the SDF and related angular distribution. After the centroid position of each particle is determined by image analysis, the positional data is fed into the software. a. Plot of the positional array with the first reference particle circled in red. b. The positional array is then aligned such that the reference particle lies on the $(0,0)$ point. c. Another particle is selected as a reference particle, the array is aligned and stacked on the previous array. $\mathbf{d}$ This process is continued until every particle has been used as a reference particle. e. A grid for the 2D histogram is defined by the user. f. The particle density per cell in the grid is calculated, which produces the spatial distribution function. g. A grid, defined in polar coordinates, is overlaid on the stacked array. $\mathbf{h}$. The particle density within each cell of the grid is determined, normalised, and then plotted in polar coordinates to provide the angular distribution.

into either the ImageJ plugin or the matlab GUI, which conducted image analysis as follows. The algorithm first selects one particle as a reference (Figure1a). It then aligns the entire positional array such that the reference particle lies at the $(0,0)$ point (Figure $1 b)$. Next, the algorithm selects the next particle as the reference, aligns the array and then stacks this array on top of the previous one (Figure 1c). This process is repeated until every particle has been used as a reference (Figure 1d). The user then chooses the range over which to calculate the density (i.e. the $\mathrm{x}$ and $\mathrm{y}$ distance from the reference particle). These values should ideally be less than one half of the size of the original image to avoid artefacts. The user provides the size of the square bin to produce a 2D-histogram of the array, which will display the spatial density of particles in relation to a reference at $(0,0)$ (Figure 1e-f). The reference particle is excluded from the SDF calculation as it would result in an intense 
peak; the resulting empty space relates to the excluded volume associated with the particles. Figure S2 (SI) demonstrates this process. Finally, the SDF is normalised by the average number of particles across the sample; this is non-trivial for finite samples. Often in RDF algorithms, a periodic boundary is applied or data around the edge of the sample is excluded to allow for accurate normalisation. Both have limitations, either through the introduction of incorrect assumptions and artefacts or the loss of valuable data. Recent studies suggested bypassing these methods by calculating the effective volume (3D) or area (2D) over which

the particles are being counted. ${ }^{25,26}$ A process inspired by this technique was implemented herein through tracking the area covered by the shifted array to normalise the SDF.

The methodology can also serve to determine the angular distribution. The same process is applied for selecting each reference particle, aligning the positional array, and stacking the data. The user then selects the radial range for investigation, Figure $1 \mathrm{~g}$. This is usually guided by the RDF, i.e. the minimum and maximum radius should correspond to the minima before and after the peak of interest, respectively. The user then provides a bin width. The angle and the particle density within each bin is then calculated and plotted in polar coordinates, Figure 1h.

\section{Results and discussion}

\section{Validation}

In order to compare the effect of increasing disorder on 2D FFT, RDF and SDF, respectively, computer generated, regular hexagonal arrays were created with a set inter-particle spacing of 100 a.u. and mean radius of 10 a.u. for each particle. Noise of a specified standard deviation ranging from $0-30 \%$ of the inter-particle spacing and particle dimensions was then added to the $\mathrm{x}, \mathrm{y}$ positions of each particle and the radius respectively. Figure 2 shows how different spatial and radius variation affect the three different analytical methods. For illustration, the SDF-based angular distribution is plotted alongside in 2e. 


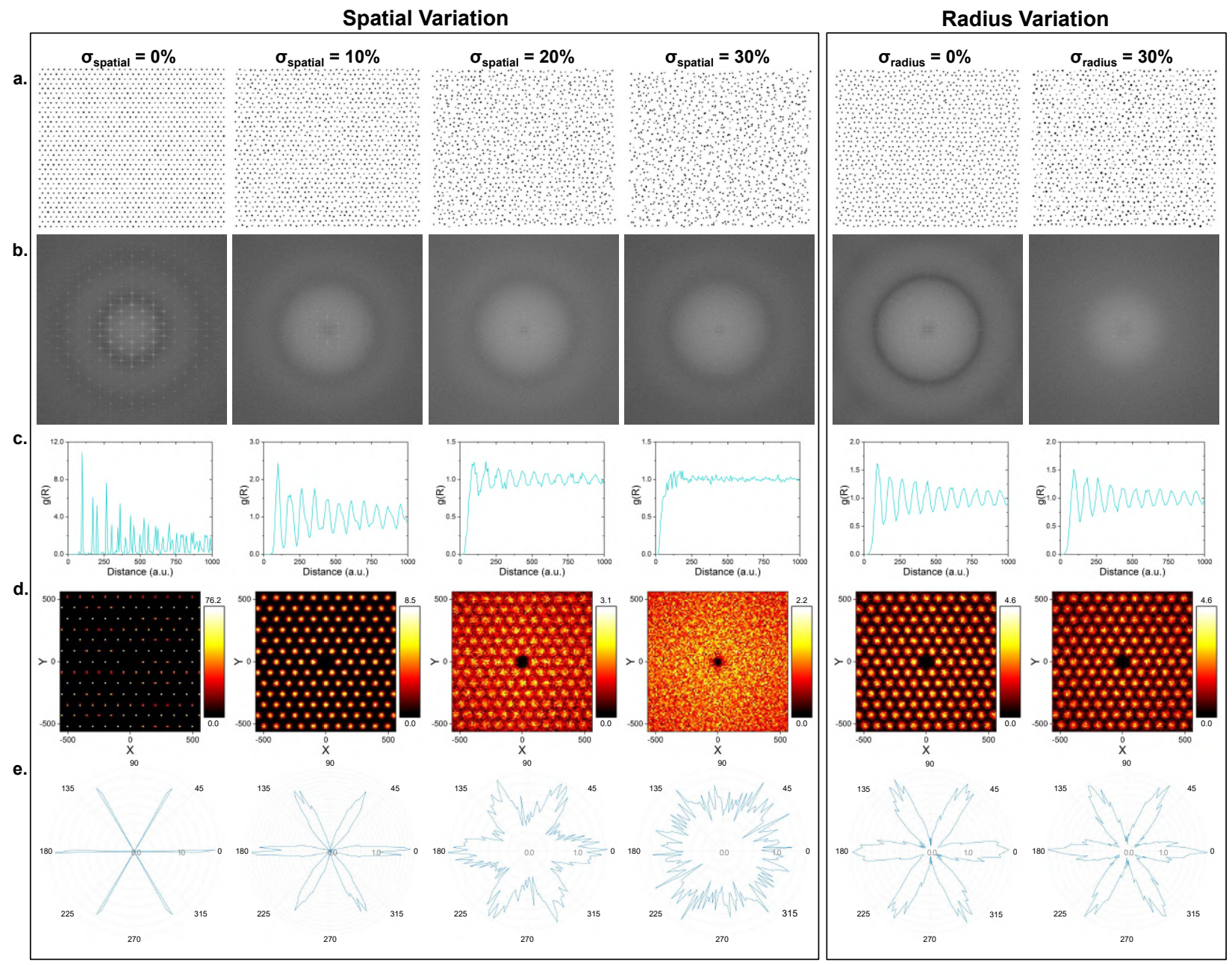

Figure 2: Validation of the SDF. On the left, the radius variation was set to $\sigma_{\text {radius }}=15 \%$ of the mean radius and the spatial variation was investigated at $\sigma_{\text {spatial }}=0$ to $30 \%$. On the right, the spatial variation $\sigma_{\text {spatial }}$ was maintained at $15 \%$ of the set inter-particle spacing and the radius variation $\sigma_{\text {radius }}$ was investigated at $0 \& 30 \%$ of the set particle radius. a. Corresponding computer generated images used for the analysis. Different analytical methods were compared: b. 2D FFT, c. RDF, d. SDF, and e. angular distribution.

On the left in Figure 2, the variation in the radius $\sigma_{\text {radius }}$ was set to $15 \%$ of the mean radius. The spatial variation $\sigma_{\text {spatial }}$ was then investigated for values of $0,10,20, \& 30 \%$ of the inter-particle spacing, respectively. The RDF at $0 \%$ showed distinct peaks characteristic of hexagonal packing; at 10 and 20\%, evidence of the hexagonal structure fades due to broadening of the peaks. For 30\%, no clear peaks of hexagonal packing could be identified. In contrast, both the SDF and 2D-FFT provided evidence of hexagonal packing up to a spatial variation of $20 \%$. At $0 \%$ both depict the high level of order, but the footprint of the 
hexagonal packing diminished more rapidly in the 2D-FFT at 10 and 20\%. For 20\%, the 2D-FFT only shows nearest neighbours, while the RDF and SDF retained more information on the ordering of the assembly for a longer range from the particle of interest. The ability of the SDF to perform well even with high levels of noise is related to how the SDF is obtained; the relative position of each particle in reference to every other particle is considered and the data is combined and normalised. This process helps to balance out random noise and allows the underlying structure and extent of order to be illustrated in the resulting SDF. In contrast, the 2D-FFT confounds positional information with particle size and shading which, while allowing the process to be entirely reversible, can increase the noise. The angular distribution (Figure 2d) is a product of the SDF, which looks at the angular dependence of the $\mathrm{n}^{\text {th }}$ neighbour, in this case the nearest neighbour. After processing the positional data to produce the SDF, a subset of the data is presented as a polar plot to produce the angular distribution. The angular distribution confirmed hexagonal packing for $0,10, \& 20 \%$ spatial variation, while any information on symmetry was lost for $30 \%$.

In a second example displayed on the right of Figure 2, the spatial variation was maintained at $15 \%$. The variation in the radius $\sigma_{\text {radius }}$ was investigated for values of $0 \& 30 \%$. It is evident that due to the nature of the RDF, SDF and angular distribution, variation in the particle radii has no effect on the outcome. The 2D-FFT is vulnerable to this variation and artefacts from the outline of the particles themselves (i.e. the pronounced ring present in the 2D-FFT in Figure 2b) impact the interpretation of the results. A full investigation on the effects of spatial and radius variation can be found in the Supporting Information (Figures S4-8).

Further validation was performed to evaluate the robustness of the SDF with respect to sample size and vacancies. It was found that all three methods were comparatively sensitive to the number of measurements used for analysis; the SDF and RDF excelled at providing the characteristic range of order while the SDF and 2D-FFT depicted the underlying structure (see Figure S9). Similar results were found for assemblies with vacancies; however, the 
SDF slightly outperformed the 2D-FFT (see Figure S10). Finally, anisotropic variation was considered (see Figure S11). Again, the extent of order was best represented by the SDF and RDF while structural information was provided by the SDF and 2D-FFT. The performance of these analytical methods was also examined for the other four 2D Bravais lattices (see Figure S12-16) with similar results.

This comparison provides justification for the validity and applicability of the SDF. When deviating from perfect ordering, it offers a more robust evaluation for both noise and underlying symmetry. With only one analytical routine, i.e. the SDF, details about the symmetry, inter-particle spacing, and the extent of ordering are revealed simultaneously in an intuitive plot. In contrast, 2D-FFT and RDF must be used in tandem and interpreted in a less straight forward fashion.

\section{Applications}

The SDF was applied to four experimental systems. Figure 3a shows an optical image of polystyrene latex particles assembled at a water-octane interface. ${ }^{19}$ This self-assembly at a liquid-liquid interface approach developed by Reimhult and coworkers allows for the formation of a non-close-packed, i.e. open structure with inter-particle spacing to be significantly above the particle diameters. Similar structures with large inter-particle separation were achieved by Law et al. ${ }^{27}$ 

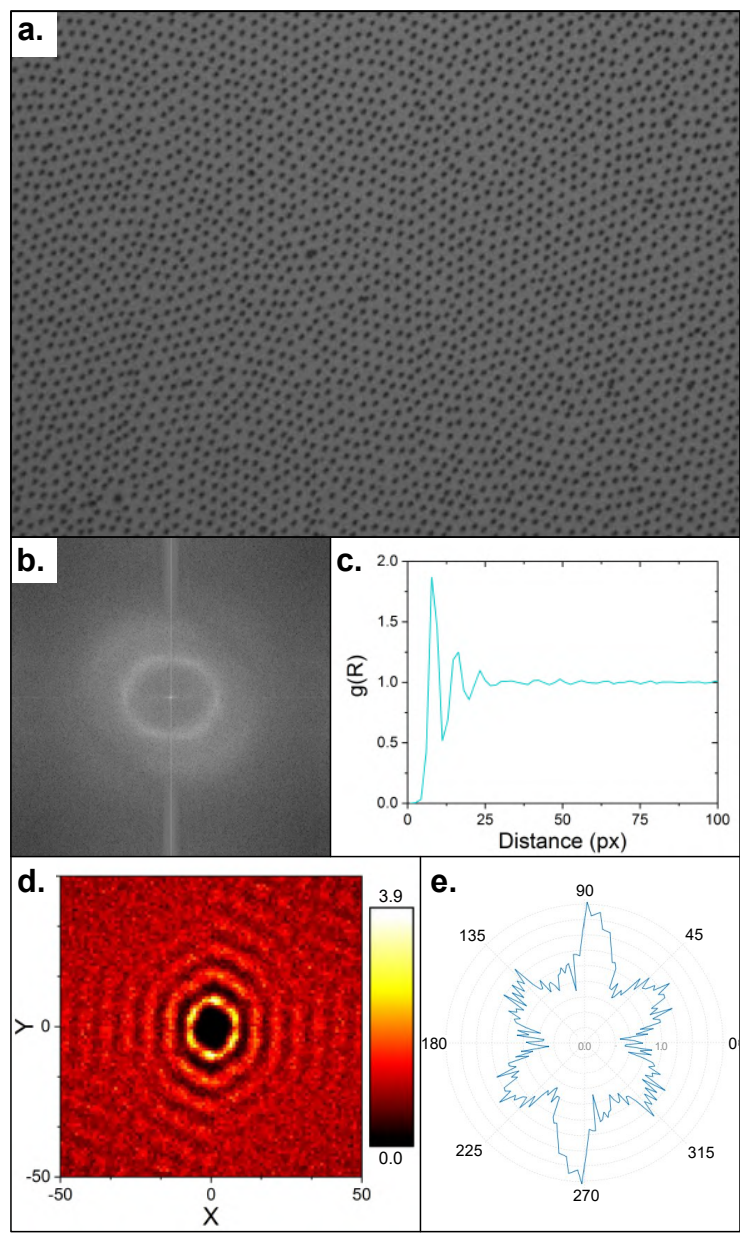

Figure 3: Polystyrene latex particles assembled at a water-octane interface a. Optical microscopic image of the assembled particles after transfer to a silicon substrate (Image width: $100 \mu \mathrm{m} ; 483 \mathrm{px}$ ), which served for analysis using different techniques: b. 2D-FFT, c. RDF, d. SDF and associated e. angular distribution. (a) Reprinted with permission from reference. ${ }^{19}$ Copyright American Chemical Society 2010.

Due to the non-closed packed nature of the assembly and associated variability in terms of spatial translation, the 2D-FFT (Figure 3b) is unable to fully resolve the hexagonal ordering. While the 2D-FFT image is indicative of more than one period of ordering, no clear evidence is provided by the image. The RDF in Figure 3c indicates 2-3 periods of ordering, which is reflected in the SDF (Figure 3d). The SDF also suggests hexagonal packing; this is confirmed by the angular distribution, which displays six broad peaks. In this example, the SDF clearly outperforms traditional methods (i.e. the combined 2D-FFT and RDF) in providing informative value of the assembly. 


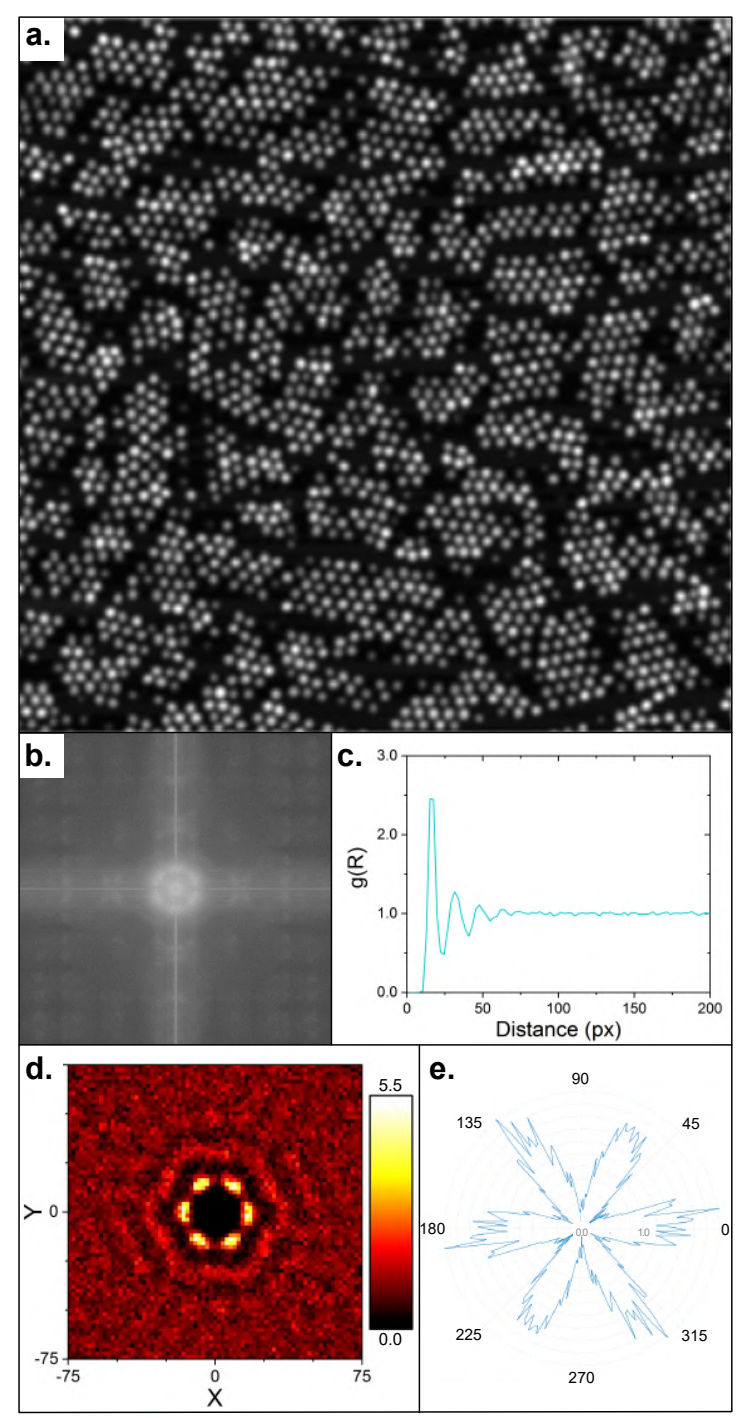

Figure 4: Microgel monolayer between transitional states. a. AFM image of the assembled particles (Image width: $10 \mu \mathrm{m} ; 822 \mathrm{px}$ ), which was analysed via: b. 2D-FFT, c. RDF, d. SDF and associated e. angular distribution. (a) Reprinted with permission from reference. ${ }^{28}$ Copyright Royal Society of Chemistry 2016.

Figure 4a shows an AFM image of a microgel monolayer in a transitional state between two crystalline phases, reprinted from a recent study by Isa and coworkers. ${ }^{28}$ Within the islands of particles there is close hexagonal packing discernible without apparent characteristic inter-island spacing or orientation. Several other studies have also shown this transition from a close packed state to an open structure. ${ }^{29,30}$ The 2D-FFT shows some evidence of the underlying hexagonal symmetry, while the RDF indicates 2-3 periods of order. In comparison, the SDF provides evidence for hexagonal packing with two well-defined periods. 
Alongside, the angle distribution for the first period confirms regular hexagonal ordering.

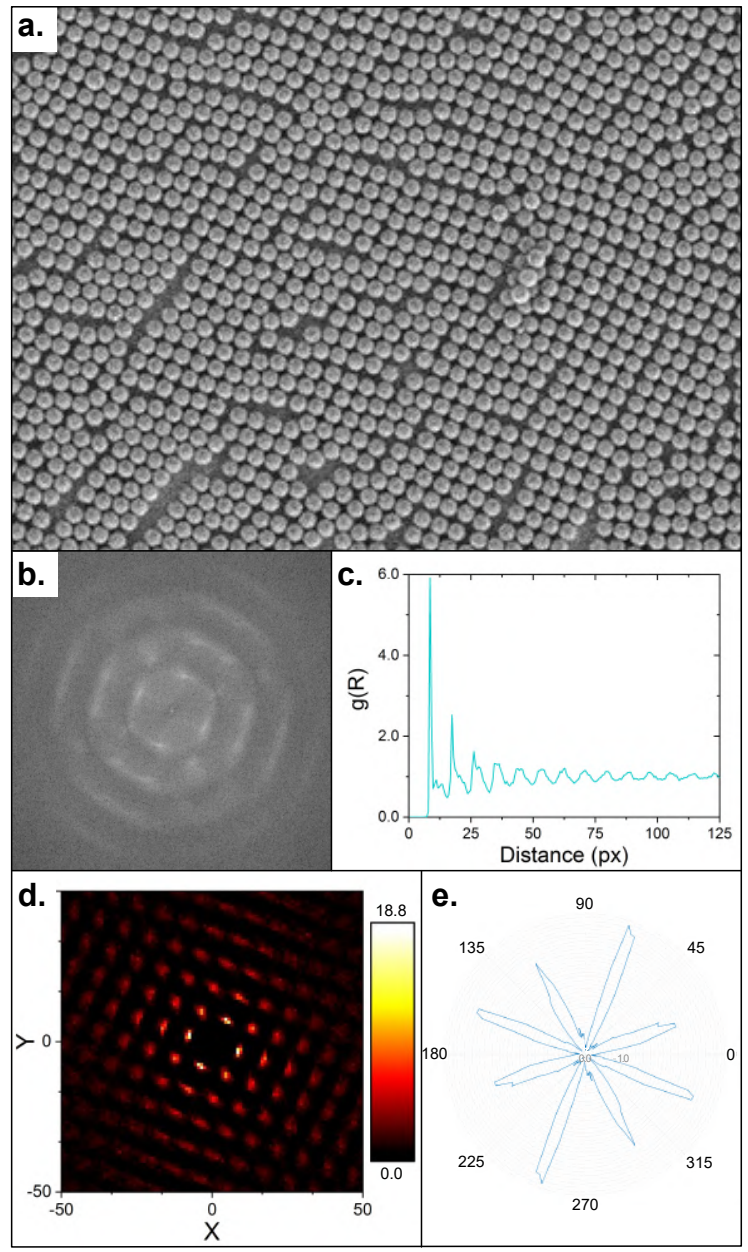

Figure 5: Polystyrene beads assembled at an air-water interface in the presence of an amphiphile. a. An SEM image of the assembled particles (Image width: $25 \mu \mathrm{m} ; 346 \mathrm{px}$ ) was analysed using different techniques: b. the 2D-FFT, c. the RDF, d. the SDF, and e. the Angular Distribution. (a) Reprinted with permission from reference. ${ }^{31}$ Copyright American Chemical Society 2018.

Typically, close packed spherical particles will form a hexagonal assembly. Vogel and coworkers achieved more complex arrays by introducing amphiphiles into a system containing polystyrene beads that were assembled at an air-water interface. ${ }^{31}$ An SEM image of a colloidal assembly achieved through this protocol is shown in Figure 5. Note that similar assemblies were attained by Hummel et al via a novel stretching protocol, which also provided access to four other 2D Bravais lattices. ${ }^{32}$ An alternative assembly with cubic symmetry was realised in binary lattices by co-assembling geometrically anisotropic colloidal particles. ${ }^{33}$ 
Image analysis shown in Figure 5b-e clearly indicates a high degree of order and square symmetry. The 2D-FFT demonstrates the underlying structure and suggests 3-4 periods of ordering. However, the regular shape of the particles and small domains of hexagonal ordering affect the 2D-FFT and interfere with the structural information. To mitigate this effect, the positional information was extracted and analysed via a separate 2D-FFT (Figure S17). While for the other examples such pre-processing did not result in significant improvements, in this case more pronounced evidence on the structural symmetry was obtained. In comparison, the RDF and SDF indicate a much higher degree of order and the SDF reproduces the cubic packing. Note that minority regions of hexagonally packed particles resulted in a slight anisotropy in the intensity of the peaks discernible by SDF. Finally, the angular distribution exhibits eight peaks, which confirms a 2D cubic packing. The width of the peaks are narrow and distinct, which provides further evidence of a high degree of ordering.

We further analysed a system exhibiting highly anisotropic ordering. Reven and coworkers exploited line defects in a smectic liquid crystal to create a unique assembly of gold nanoparticles. ${ }^{34} \mathrm{~A}$ solution of gold nanoparticles in $8 \mathrm{CB}$ was placed in a wedge cell, which induced linear alignment of nanoparticle aggregates. This type of structure has been observed in other experimental systems such as that of Müller et al., who deposited nanoparticles onto the wrinkled surface of pre-stretched PDMS. ${ }^{35}$ 


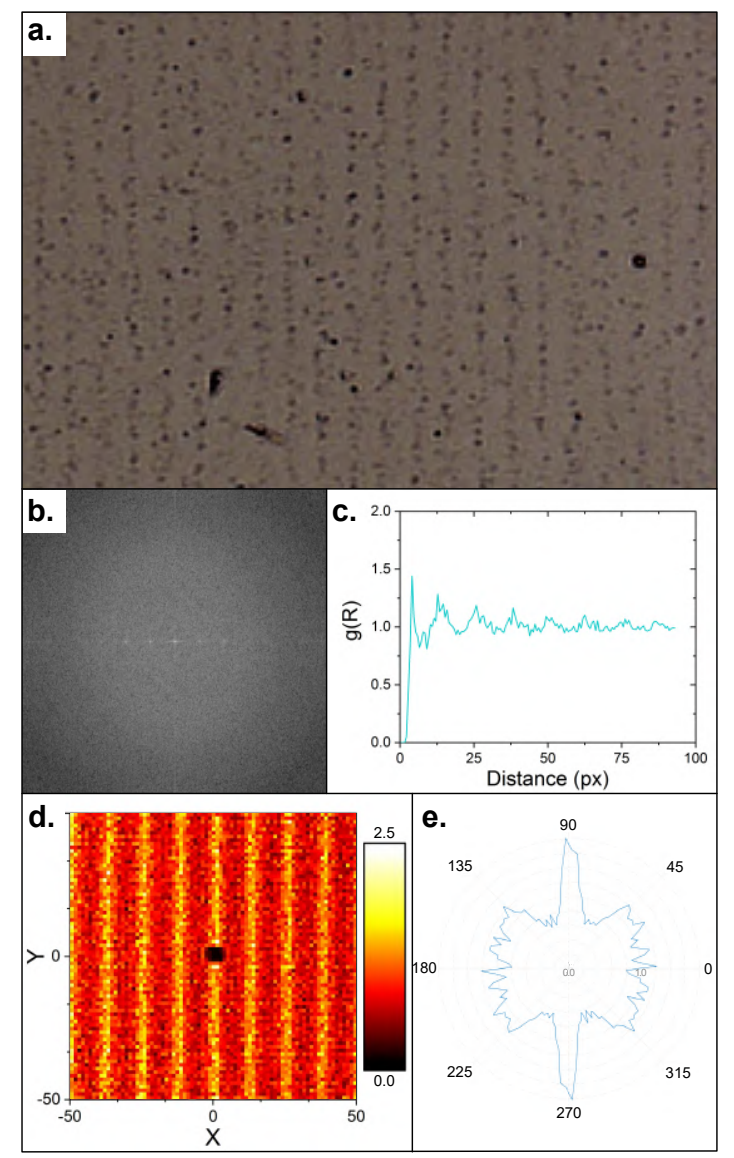

Figure 6: Gold nanoparticles in a smectic liquid crystal. a. Polarized optical microscopy (POM) image of the composite inside a homeotropic wedge cell (Image width: $190 \mu \mathrm{m} ; 274 \mathrm{px}$ ), which was analysed via: b. 2D-FFT, c. RDF, d. the SDF and associated e. angular distribution. (a) Reprinted with permission from reference. ${ }^{34}$ Copyright Royal Society of Chemistry 2012.

The assembly shown in Figure 6a is unique in in the fact that there is no characteristic inter-particle distance within each line defect but a periodic arrangement of the line defects themselves. The 2D-FFT is able to show up to 5 periods of the line defects (two on either side of the central peak) while the RDF shows some evidence of order. In comparison, the $\mathrm{SDF}$ is able to more accurately reproduce the extent of the ordering of the system. The angular distribution for this system reflects the anisotropy (here conducted for a range of 0-20 px). Along the direction of the defect, an increase in the particle density can be seen while perpendicular to the defect, this density reduces to the bulk value.

In conclusion, we provide a comprehensive evaluation on the use of SDF to assess order- 
ing in colloidal monolayers. Systematic variation of inter-particle spacing, diameter, total population and monolayer coverage as well as the application to four prominent examples in the literature reveal that a) the SDF is at least as successful as the RDF in determining the orderliness of an assembly; and b) the SDF is equally capable of demonstrating the underlying symmetry when compared to the 2D-FFT. Experimental data for less highly ordered systems confirms the RDF fails at providing information about structure in non-crystalline systems. The 2D-FFT rapidly looses information about the extent of the order and, at times, could not determine the underlying symmetry for less ordered assemblies. In contrast, the $\mathrm{SDF}$ is able to retain information regarding the structure and the extent of the order while providing an accurate representation of the ordering in the assembly in real space. With our accompanying imageJ plugin and standalone software, the implementation of the SDF is straightforward and should provide a valuable addition in the toolbox of monolayer characterisation.

\section{Acknowledgement}

NMF is grateful for the EPSRC for a Doctoral Training Partnership (EP/M507970/1).

\section{Supporting Information Available}

The following files are available free of charge.

- Supplementary Information: In depth methodology, figure of image analysis, figure demonstrating methodology; full validation including generation of test arrays, 2DFFT, RDF, SDF, and angular distribution for spatial and radius variation from 0$30 \%$; sample size sensitivity analysis; vacancy sensitivity analysis; anisotropic ordering; demonstration of the SDF and angular distribution and comparison with the 2D-FFT and RDF for the remaining 4 Bravais lattices. 
- ImageJ Plugin

- Matlab Code

- Standalone Software for Windows

- Standalone Software for Mac

\section{References}

(1) Lotito, V.; Zambelli, T. Approaches to self-assembly of colloidal monolayers: A guide for nanotechnologists. Advances in Colloid and Interface Science 2017, 246, 217-274.

(2) Horcas, I.; Fernandez, R.; Gomez-Rodriguez, J. M.; Colchero, J.; Gomez-Herrero, J.; Baro, A. M. WSXM: A software for scanning probe microscopy and a tool for nanotechnology. Review Of Scientific Instruments 2007, 78, 013705(1-8).

(3) Schneider, C. A.; Rasband, W. S.; Eliceiri, K. W. NIH Image to ImageJ: 25 years of image analysis. Nature Methods 2012, 9, 671-675.

(4) Brocher, J. Qualitative and Quantitative Evaluation of Two New Histogram Limiting Binarization Algorithms. International Journal of Image Processing 2014, 8, 30-48.

(5) Gonzales, R. C.; Woods, R. E. Digital Image Processing, 2nd ed.; Prentice Hall: New Jersey, 2002; Chapter 4, pp 147-212.

(6) Bigioni, T. P.; Lin, X.-M.; Nguyen, T. T.; Corwin, E. I.; Witten, T. A.; Jaeger, H. M. Kinetically driven self assembly of highly ordered nanoparticle monolayers. Nature Materials 2006, 5, 265-270.

(7) Toolan, D. T. W.; Fujii, S.; Ebbens, S. J.; Nakamura, Y.; Howse, J. R. On the mechanisms of colloidal self-assembly during spin-coating. Soft Matter 2014, 10, 8804-8812. 
(8) Bai, L.; Mai, V. C.; Lim, Y.; Hou, S.; Möhwald, H.; Duan, H. Large-Scale Noniridescent Structural Color Printing Enabled by Infiltration-Driven Nonequilibrium Colloidal Assembly. Advanced Materials 2018, 30, 1705667(1-7).

(9) Rupich, S. M.; Castro, F. C.; Irvine, W. T. M.; Talapin, D. V. Soft epitaxy of nanocrystal superlattices. Nature Communications 2014, 5, 5045(1-10).

(10) Talapin, D. V.; Shevchenko, E. V.; Bodnarchuk, M. I.; Ye, X.; Chen, J.; Murray, C. B. Quasicrystalline order in self-assembled binary nanoparticle superlattices. Nature $\mathbf{2 0 0 9}$ 461, 964-967.

(11) Chen, Q.; Bae, S. C.; Granick, S. Directed self-assembly of a colloidal kagome lattice. Nature 2011, 469, 381-384.

(12) Meder, F.; Thomas, S. S.; Bollhorst, T.; Dawson, K. A. Ordered Surface Structuring of Spherical Colloids with Binary Nanoparticle Superlattices. Nano Letters 2018, 18, $2511-2518$.

(13) Kirkwood, J. G.; Boggs, E. M. The Radial Distribution Function in Liquids. The Journal of Chemical Physics 1942, 10, 394-402.

(14) Choi, Y.; Ree, T.; Ree, F. H. Hard-sphere radial distribution functions for face-centered cubic and hexagonal close-packed phases: Representation and use in a solid-state perturbation theory. The Journal of Chemical Physics 1991, 95, 7548-7561.

(15) Yang, H.; Jiang, P. Large-Scale Colloidal Self-Assembly by Doctor Blade Coating. Langmuir 2010, 26, 13173-13182.

(16) Zhang, J.-T.; Wang, L.; Lamont, D. N.; Velankar, S. S.; Asher, S. A. Fabrication of Large-Area Two-Dimensional Colloidal Crystals. Angewandte Chemie 2012, 124, 62216224 . 
(17) Lotito, V.; Zambelli, T. Self-Assembly of Single-Sized and Binary Colloidal Particles at Air/Water Interface by Surface Confinement and Water Discharge. Langmuir 2016, 32, 9582-9590.

(18) Kumar, A.; Kumar Mandal, B.; Mishra, P. Morphology of Colloidal Particles Dispersed in Nematic Solvent. Journal of Physics: Conference Series 2016, 765, 012022(1-7).

(19) Isa, L.; Kumar, K.; Müller, M.; Grolig, J.; Textor, M.; Reimhult, E. Particle Lithography from Colloidal Self-Assembly at Liquid-Liquid Interfaces. ACS Nano 2010, 4, $5665-5670$.

(20) Brodin, A.; Nych, A.; Ognysta, U.; Lev, B.; Nazarenko, V.; Škarabot, M.; Muševič, I. Condensed matter physics; Institute for Condensed Matter Physics of the National Academy of Sciences of Ukraine, 1994.

(21) Harun-Ur-Rashid, M.; Bin Imran, A.; Seki, T.; Ishii, M.; Nakamura, H.; Takeoka, Y. Angle-Independent Structural Color in Colloidal Amorphous Arrays. ChemPhysChem 2010, 11, 579-583.

(22) Svishchev, I. M.; Kusalik, P. G. Structure in liquid water: A study of spatial distribution functions. The Journal of Chemical Physics 1993, 99, 3049-3058.

(23) Kusalik, P. G.; Svishchev, I. M. The spatial structure in liquid water. Science (New York, N.Y.) 1994, 265, 1219-1221.

(24) Hata, T.; Ono, Y. Difference Spatial Distribution Function Analysis of Methanol and Ethanol Solutions. Chemical \&3 Pharmaceutical Bulletin 1999, 47, 615-620.

(25) Larsen, M. L.; Shaw, R. A. A method for computing the three-dimensional radial distribution function of cloud particles from holographic images. Atmospheric Measurement Techniques 2018, 11, 4261-4272. 
(26) Kopera, B. A. F.; Retsch, M. Computing the 3D Radial Distribution Function from Particle Positions: An Advanced Analytic Approach. Analytical Chemistry 2018, 90, $13909-13914$.

(27) Law, A. D.; Buzza, D. M. A.; Horozov, T. S. Two-Dimensional Colloidal Alloys. Physical Review Letters 2011, 106, 128302(1-4).

(28) Rey, M.; Fernández-Rodríguez, M. Á.; Steinacher, M.; Scheidegger, L.; Geisel, K.; Richtering, W.; Squires, T. M.; Isa, L. Isostructural solid-solid phase transition in monolayers of soft core-shell particles at fluid interfaces: structure and mechanics. Soft Matter 2016, 12, 3545-3557.

(29) El-Tawargy, A. S.; Stock, D.; Gallei, M.; Ramadan, W. A.; Shams El-Din, M. A.; Reiter, G.; Reiter, R. Multiple Structural Transitions in Langmuir Monolayers of Charged Soft-Shell Nanoparticles. Langmuir 2018, 34, 3909-3917.

(30) Rey, M.; Hou, X.; Tang, J. S. J.; Vogel, N. Interfacial arrangement and phase transitions of PNiPAm microgels with different crosslinking densities. Soft Matter 2017, 13, 87178727.

(31) Rey, M.; Yu, T.; Bley, K.; Landfester, K.; Buzza, D. M. A.; Vogel, N. AmphiphileInduced Anisotropic Colloidal Self-Assembly. Langmuir 2018, 34, 9990-10000.

(32) Hummel, M. E. J.; Stelling, C.; Kopera, B. A. F.; Nutz, F. A.; Karg, M.; Retsch, M.; Förster, S. Ordered Particle Arrays via a Langmuir Transfer Process: Access to Any Two-Dimensional Bravais Lattice. Langmuir 2019, 35, 973-979.

(33) Paik, T.; Diroll, B. T.; Kagan, C. R.; Murray, C. B. Binary and Ternary Superlattices Self-Assembled from Colloidal Nanodisks and Nanorods. Journal of the American Chemical Society 2015, 137, 6662-6669. 
(34) Milette, J.; Relaix, S.; Lavigne, C.; Toader, V.; Cowling, S. J.; Saez, I. M.; Lennox, R. B.; Goodby, J. W.; Reven, L. Reversible long-range patterning of gold nanoparticles by smectic liquid crystals. Soft Matter 2012, 8, 6593-6598.

(35) Müller, M.; Karg, M.; Fortini, A.; Hellweg, T.; Fery, A. Wrinkle-assisted linear assembly of hard-core/soft-shell particles: impact of the soft shell on the local structure. Nanoscale 2012, 4, 2491-2499.

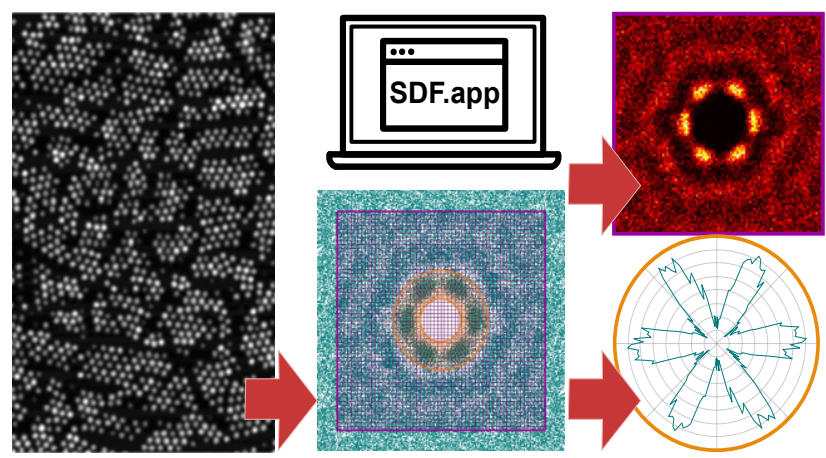

For Table of Contents Only. 